\title{
Manipulation of cells' position across a microfluidic channel using a series of continuously varying herringbone structures
}

\author{
Yugyung Jung ${ }^{1}$, Ji-chul Hyun ${ }^{2}$, Jongchan Choi ${ }^{2}$, Arslan Atajanov ${ }^{1}$ and Sung Yang ${ }^{1,2^{*}}$
}

\begin{abstract}
Controlling cells' movement is an important technique in biological analysis that is performed within a microfluidic system. Many external forces are utilized for manipulation of cells, including their position in the channel. These forces can effectively control cells in a desired manner. Most of techniques used to manipulate cells require sophisticated set-ups and equipment to generate desired effect. The exception to this is the use of hydrodynamic force. In this study, a series of continuously varying herringbone structures is proposed for positioning cells in a microfluidic channel using hydrodynamic force. This structure was experimentally developed by changing parameters, such as the length of the herringbone's apex, the length of the herringbone's base and the ratio of the height of the flat channel to the height of the herringbone structure. Results of this study, have demonstrated that the length of the herringbone's apex and the ratio of the heights of the flat channel and the herringbone structure were crucial parameters influencing positioning of cells at $100 \mu \mathrm{l} / \mathrm{h}$ flow rate. The final design was fixed at 170 and $80 \mu \mathrm{m}$ for the length of herringbone's apex and the length of herringbone's base, respectively. The average position of cells in this device was $34 \mu \mathrm{m}$ away from the side wall in a $200 \mu \mathrm{m}$ wide channel. Finally, to substantiate a practical application of the herringbone structure for positioning, cells were randomly introduced into a microfluidic device, containing an array of trapping structures together with a series of herringbone structures along the channel. The cells were moved toward the trapping structure by the herringbone structure and the trapping efficiency was increased. Therefore, it is anticipated that this device will be utilized to continuously control cells' position without application of external forces.
\end{abstract}

Keywords: Positioning, Series of herringbone structure, Hydrodynamic force

\section{Background}

In the last decade, fast and cost-effective manipulation of the movement of cells has been under the spotlight of research in the field of microfluidics and biomedicine. Novel methods and devices for positioning and subsequent separation of cells are used in the medical environment for on-chip analysis of human cells [1]. Some benefits of using microfluidics in cellular analyses include high-throughput screening, precise manipulation of the fluid flow, minimal reagent consumption and high sensitivity to small amounts of a sample. In addition,

\footnotetext{
*Correspondence: syang@gist.ac.kr

${ }^{1}$ Department of Biomedical Science and Engineering, Gwangju Institute of Science and Technology (GIST), Gwangju 61005, Republic of Korea Full list of author information is available at the end of the article
}

small form-factor allows integration with other technologies to enable even broader spectrum of functionalities. Researchers were able to achieve individual cell capture using various microstructures, such as cup-shapes and grooves $[1,4,5]$. The fact that this is possible to achieve using only manipulation of hydrodynamic forces by changing the geometry of the channel has created hopes for wider commercialization opportunities for microfluidic systems and accelerated research efforts in this field. Microstructure-based fluid flow manipulation is often used for several applications, e.g. cell sorting, bioreactors, single cell analysis, drug discovery chips and tissue engineering [3].

Cell manipulation in a microfluidic system can be realized using various methods, among them are optical, electrical, magnetic and hydrodynamic. 
Optical manipulation of cells is enabled by a technique called optical trapping. This technique enables trapping of cells using a system consisting of a microscope with a high numerical aperture objective lens and a highlyfocused laser beam, which exerts optical forces on cells or particles. These forces enable optical trapping. However, the use of highly focused laser beam exposes cells to photodamage or heat damage. For example, the use of light in the UV range will cause mutations that could have an adverse effect on the cell and subsequent analyses. Wavelength of the beam can be manipulated to avoid photodamage. All in all, optical manipulation is a promising tool for a contactless nano scale manipulation and measurement in the field of cell biology $[1,3]$.

Electrical manipulation of cells is carried out mostly using a technique called dielectrophoresis (DEP). This technique uses varying dielectric properties of media and particles or cells when exposed to a non-uniform electric field. The effect is most prominent in particles with the largest polarity of charges. The DEP technique has been a useful and commercially applicable tool in microfluidics by enabling robust non-invasive positioning, separation and trapping of cells and particles. The major disadvantages of this method include, joule heating and modification of transmembrane potential, which could lead to cell damage and other undesirable effects on the sample's integrity $[1,3]$.

Magnetic manipulation of cells is enabled by utilizing intrinsic or extrinsic factors. In the intrinsic magnetic manipulation technique, magnetic properties of iron-containing cells and biomolecules are harnessed to enable manipulation. On the other hand, extrinsic magnetic manipulation technique uses cells conjugated with magnetically active external components, such as magnetic nanoparticles to enable manipulation. Similarly, to other cell manipulation techniques, magnetic manipulation allows contactless positioning, sorting and trapping of cells and particles. Among the disadvantages of the magnetic technique is use of magnetic beads for labelling $[1,3]$.

Hydrodynamic manipulation of cells in a microfluidic environment is achieved by utilizing geometry of channels and properties of fluids. First examples of such manipulation harnessed capillary effect, however the development of microfabrication technologies enabled more sophisticated ways of hydrodynamic manipulation of cells and fluid flow. As an example, cells could be aligned to a single line by using sheath flow, this technique is one of many alternative hydrodynamic focusing techniques $[1,2]$.

One of the techniques extensively studied in the recent years is deterministic lateral displacement (DLD). This technique uses an array of structures located under a certain angle to the main channel to separate cells or particles based on their size. The structure of the DLD array can be of different geometry, each of which exhibits differing effects on the cell or particle flow in the fluid. The major factor that determines performance and a desired effect of the DLD array is the critical diameter of the streamlines [6].

An alternative method for cell positioning was proposed by Rhee et al. [4], which used centrifugal forces to move and position cells inside a microchannel. The authors noted that this method did not have an adverse effect on the viability of cells and the main advantage of this method is the ability to control the density of positioned cells by changing the density of the cell suspension. However, this method has considerable disadvantages, e.g. cells that exhibit anchoring properties are to be avoided due to the rapid and firm attachment to structures and each other. The other disadvantage is the lack of tunability of the desired outcome, i.e. many variables need to be controlled to achieve results which is itself limited to a certain position at the interface with a microchannel wall [4].

The hydrodynamic systems can exhibit clogging and cell damage by high shear stress. In this study, we suggest an improved herringbone structure for manipulation of the position of cells along the channel in a microfluidics device. To support the enhanced properties of the herringbone structure proposed herein we apply it to attempt to improve cell trapping efficiency $[1,2,4-6,8$, 9].

\section{Methods}

\section{Fabrication and schematics of the device}

Microfluidic devices were fabricated by photolithography and soft lithography techniques. For patterning of structures on silicon wafers (100) SU8 2010 and SU8 2025 (Microchem Corp.) were used to coat bottom and top layers. A simple flat channel was patterned on the bottom layer and herringbone structures were patterned on the top layer. The width of the channel is $200 \mu \mathrm{m}$. The heights of the bottom and top layers are 18 and $27 \mu \mathrm{m}$, respectively. Noteworthy, the height ratio between the flat channel and the herringbone structure is 1.5.

To form polydimethylsiloxane (PDMS) cast, a conventional soft lithography method was performed. PDMS (Sylgard 184, Dow Corning, Korea) was mixed with a curing agent at a 10:1 ratio and poured onto the master. After degassing, it was cured for $1 \mathrm{~h} 30 \mathrm{~min}$ at $75^{\circ} \mathrm{C}$. Cured PDMS mold was peeled off from the master and each device was separated by cutting. The inlet and outlet holes were made in the top layer. Then the top and 
bottom layers were exposed to oxygen plasma (Femto Science, Korea) for $1 \mathrm{~min}$ and precisely attached to each other using contact micro-aligner (MIDAS, Korea). The graphical representation of the device can be seen in Fig. 1.

\section{Preparation of sample}

Experiments were performed with polystyrene fluorescent particles (diameter $10.2 \mu \mathrm{m}, 1 \%$ concentration $(\mathrm{v} / \mathrm{v})$, i.e. $2.25 \times 10^{6}$ particles $/ \mathrm{ml}$ from Spherotech Inc.) and with Jurkat cells (Jurkat clone E6-1). Jurkat cells are

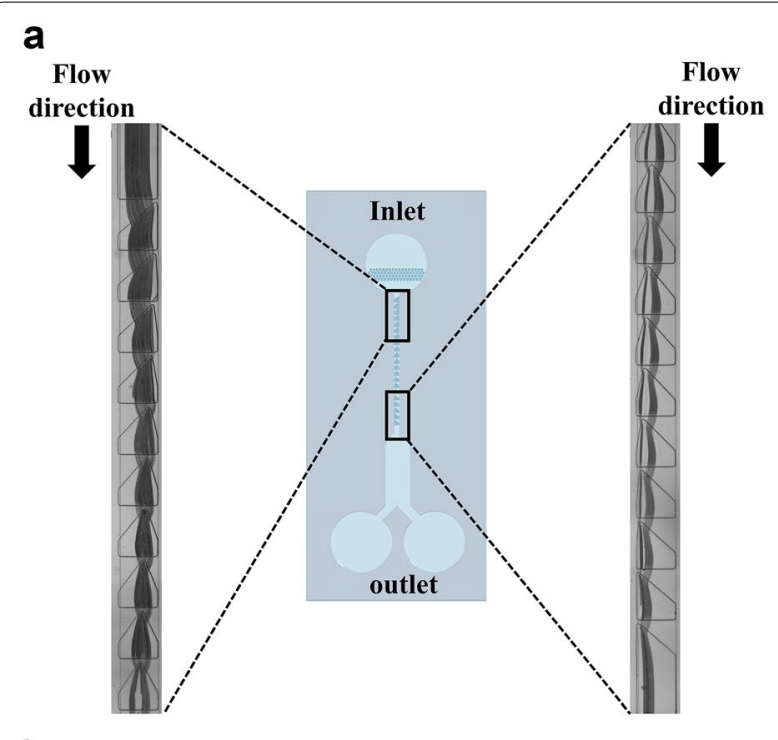

b

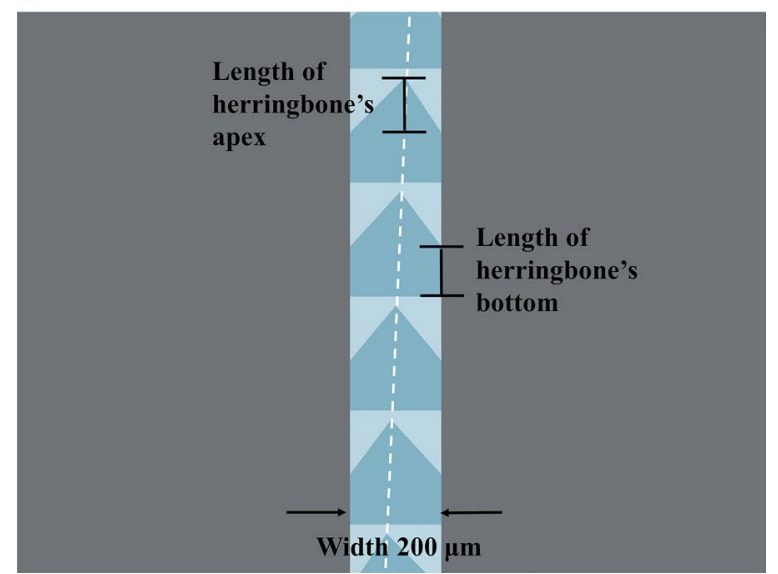

Fig. 1 a Schematic of the herringbone structure in the microfluidic device which consists of two layers, an inlet and two outlets. One layer is a simple flat channel (bottom layer) and the other layer has a herringbone structure (top channel). b The herringbone structure's apex moves from right side to left side gradually 10 by $10 \mu \mathrm{m}$ (white line) and the total length of the structure itself is comprised of the length of herringbone's apex and length of herringbone's base human leukemia T-cells. The cells were obtained from Korean cell line bank.

The polystyrene particles were diluted from $2.25 \times 10^{6}$ particles $/ \mathrm{ml}$ to $2.25 \times 10^{5}$ particles $/ \mathrm{ml}$ concentration with $0.05 \%$ tween 20 solution. Jurkat cell sample was diluted in phosphate buffered saline (PBS) to a concentration of approximately $5.7 \times 10^{5} \mathrm{cells} / \mathrm{ml}$.

\section{Experimental set up and analysis method}

A high-speed camera (Motionpro X3, Redlake) was used for tracking the $x$-position of particles at the inlet region and at the last herringbone structure. $X$-position represents the distance from the apex to the left side wall. The camera was set to capture 500 frames with a frequency of $800 \mathrm{~Hz}$. The sample fluid was injected by a syringe pump (neMESYS, CentoniGmbH, Germany) at $100 \mu \mathrm{l} / \mathrm{h}$ flow rate.

Particles' $x$-positions at the last herringbone structure were analyzed using ImageJ software (National Institutes of Health). As shown in Fig. 2a-b, the background was removed from the original image using auto-threshold function. Now the image consisted of only 0 or $255 \mathrm{val}-$ ues indicating presence and absence of the signal, respectively. Then, by setting a sampling line across the channel on processed images, we were able to extract particles' position expressed in a plot as peaks of signal. The peaks indicate boundaries of a particle. From the data, particles' $x$-positions within the channel was collected.

\section{Results and discussion}

\section{Working principle of the device and numerical study}

Particles, introduced from the inlet, have random positions across the channel. However, upon reaching the region with a series of herringbone structures they are continuously positioned closer to the apex of each herringbone structure. The positioning of particles is caused by the additional pressure gradient generated by each herringbone structure. As shown in Fig. 3a, the apex is a low-pressure region and in Fig. 3b the opposite is observed, the center is a high-pressure region. Noteworthy, magnitude of the low pressure at the apex ranges from 141 to $145 \mathrm{~Pa}$ and the magnitude of the high pressure at the base ranges from 118 to $119 \mathrm{~Pa}$. In other words, particles are sequentially focused and dispersed, while they travel through the channel. Each individual herringbone structure's apex is displaced by $10 \mu \mathrm{m}$ toward the left side wall. Thus, by the end of the series particles' flow near the left side wall.

This study attempts to increase the focusing pressure and decrease the dispersing pressure to achieve accurate particle positioning by changing the herringbone structure's geometry. 


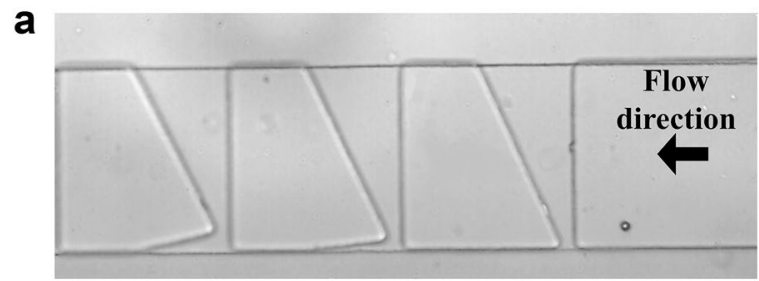

A

b

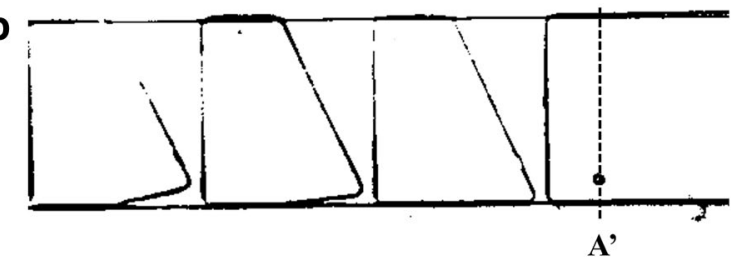

C

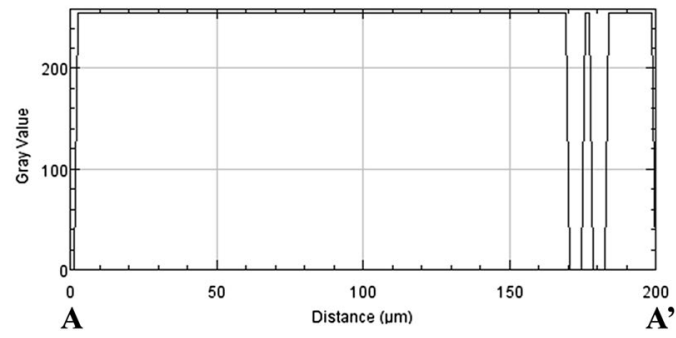

Fig. 2 a-c Image analysis of particles in the inlet region using ImageJ software. a Original image taken from the high-speed camera. b Processed image after conversion to gray scale for removal of the background. c Measurement of the particle's position across the channel. The peaks indicate particle's boundaries

\section{Experimental study}

In this study, polystyrene fluorescent beads with a diameter of $10.2 \mu \mathrm{m}$ and concentration of $0.1 \%$ were introduced into the device at $100 \mu \mathrm{l} / \mathrm{h}$ flow rate. The first experiment was performed using microfluidic devices with various lengths of herringbone's apex: 90, 130, 170 and $210 \mu \mathrm{m}$. Depending on the length of herringbone's apex, the particles had different average $x$-position. The device with $90 \mu \mathrm{m}$ length of the apex had particles positioned at the average of $81 \mu \mathrm{m}$ away from the wall and standard deviation was larger than in others. On the other hand, the device with $170 \mu \mathrm{m}$ length of the apex had the best performance compared to other devices. Increase of the apex's length resulted in a more accurate positioning of particles. This is due to the effect of the slope of the apex, the sharper slope of the apex leads to a stronger pressure drop yielding particle focusing. In addition, the pressure gradient region is extended together with the extension of the length of the apex, thus particles experience hydrodynamic forces over a longer period of time. In the case of $210 \mu \mathrm{m}$, the particles' $x$-position wasn't decreased further. No statistically significant difference in the average $x$-position of cells between devices with the 170 and $210 \mu \mathrm{m}$ apex length was measured. It is inferred that particle's $x$-position does not decrease after a certain point despite further increase in the length of the herringbone's apex (Fig. 4a).

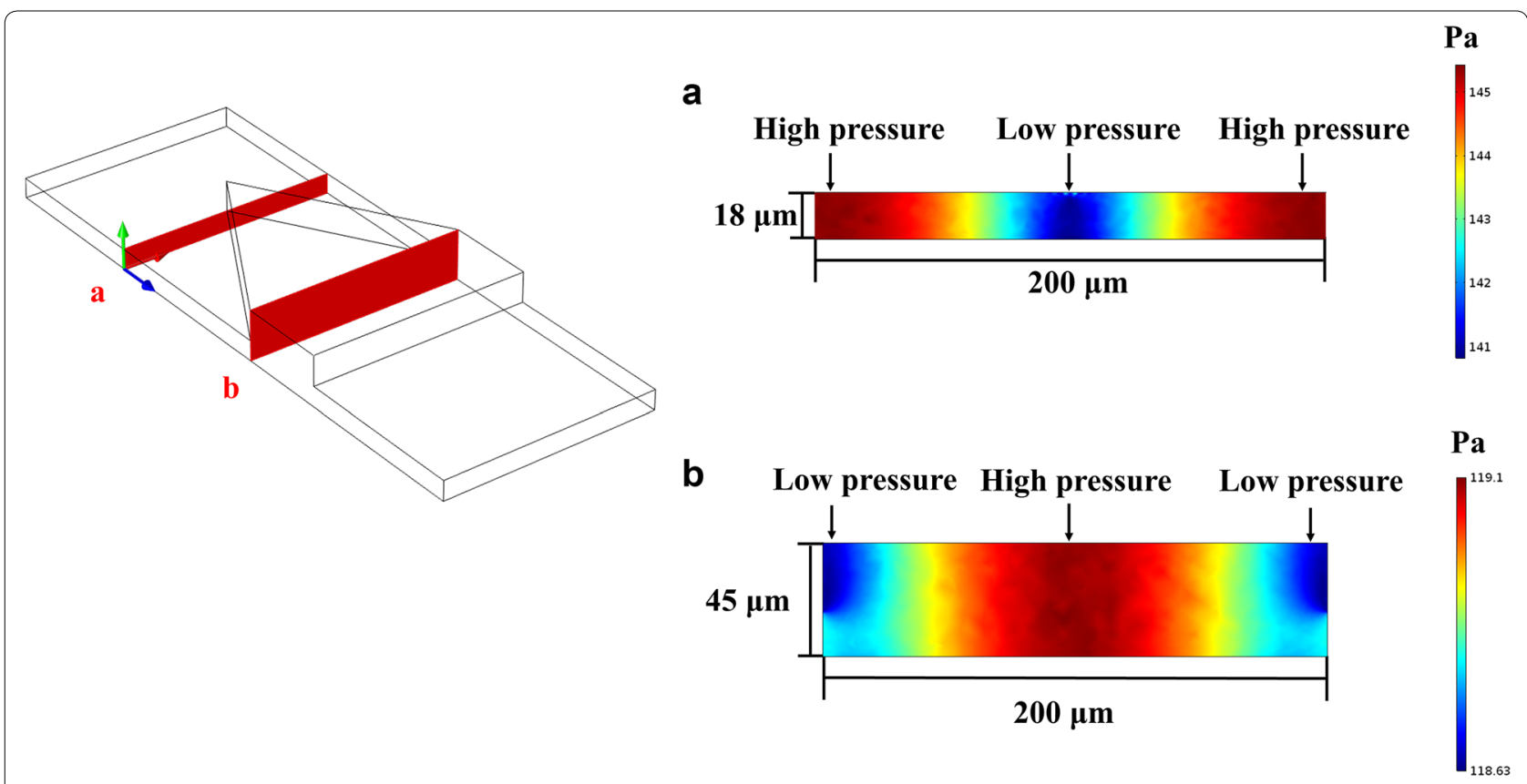

Fig. 3 Pressure distribution pattern at the vicinity of the herringbone structure. a Pressure distribution at the apex of the herringbone structure. b Pressure distribution at the origin of apex extrusion 
Further, various lengths of the herringbone's base were experimentally tested. They are 20,50, 80 and $110 \mu \mathrm{m}$. As shown in Fig. 4b, there is a slight difference in the average value of $x$-position of the focused stream. However, there were no statistically significant differences. As a result, the length of the herringbone's base is not a major factor affecting efficiency of particle positioning.

Lastly, the ratios between the height of the flat channel and the herringbone structure was tested. The flat channel's height was fixed at $18 \mu \mathrm{m}$. Herringbone structures' heights had 1:0.5, 1:1, 1:1.5, 1:2 ratios to the flat channel height. Experimental results, provided in Fig. 5, demonstrate the effect of the ratio on positioning accuracy. Particles were positioned closer to the side wall in devices with 1:1.5 and 1:2 ratios. Thus, it was concluded that the increased height of the herringbone structure results in a more prominent positioning effect. This is due to the change in the pressure drop in the region of the herringbone structure. Height of the herringbone structure is directly proportional to the hydrodynamic force exerted on particles. Further, it was found that the effect of the heights ratio made little difference beyond the ratio of 1:1.5.

The device, used in the final experiment, was designed with consideration of the experimental results. The device had the following parameters: length of herringbone's apex $-170 \mu \mathrm{m}$, height of herringbone's base $-80 \mu \mathrm{m}$ and height ratio of the flat channel to the herringbone structure-1:1.5. Samples used in the experiment contained fluorescent beads and Jurkat cells. They were used to measure positioning effect across the channel's width at each flow rate, i.e. 100, 500 and $1000 \mu \mathrm{l} / \mathrm{h}$. Statistical analysis has revealed that there is

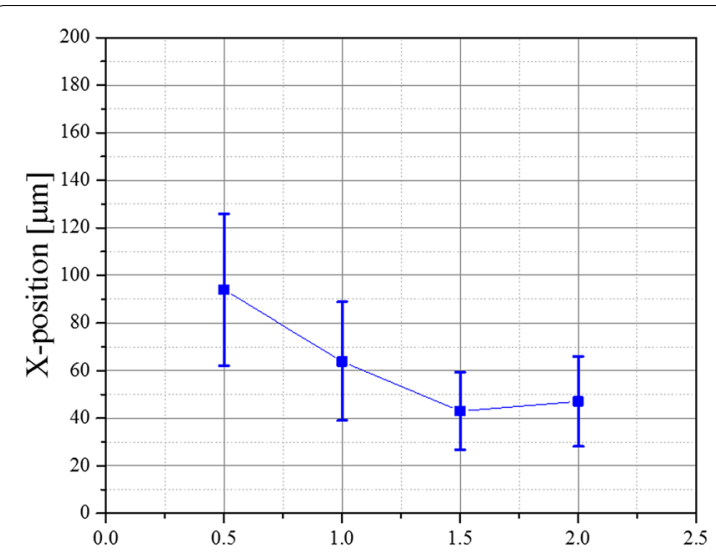

Height ratio of the flat channel to the herringbone structure

Fig. 5 The result of particle positioning across the channel as a function of the height ratio between the flat channel and the herringbone structure. $X$ axis and $Y$ axis represent the ratios and particles' average $x$-position across the channel

no significant difference in the positioning cells and particles at various flow rates (Fig. 6). From the final experiment result, it was concluded that cells can be effectively positioned in a desired region across a microfluidic channel using hydrodynamic forces brought about by a series of continuously changing herringbone structures. Although the cells' average position did not reach the herringbone's apex precisely, cells were positioned at the vicinity of herringbones' apex. The length of herringbones' apex and ratio of the flat channel to the herringbone structure are important parameters that underpin the improved performance of the proposed structure. The performance is defined by the $x$-position
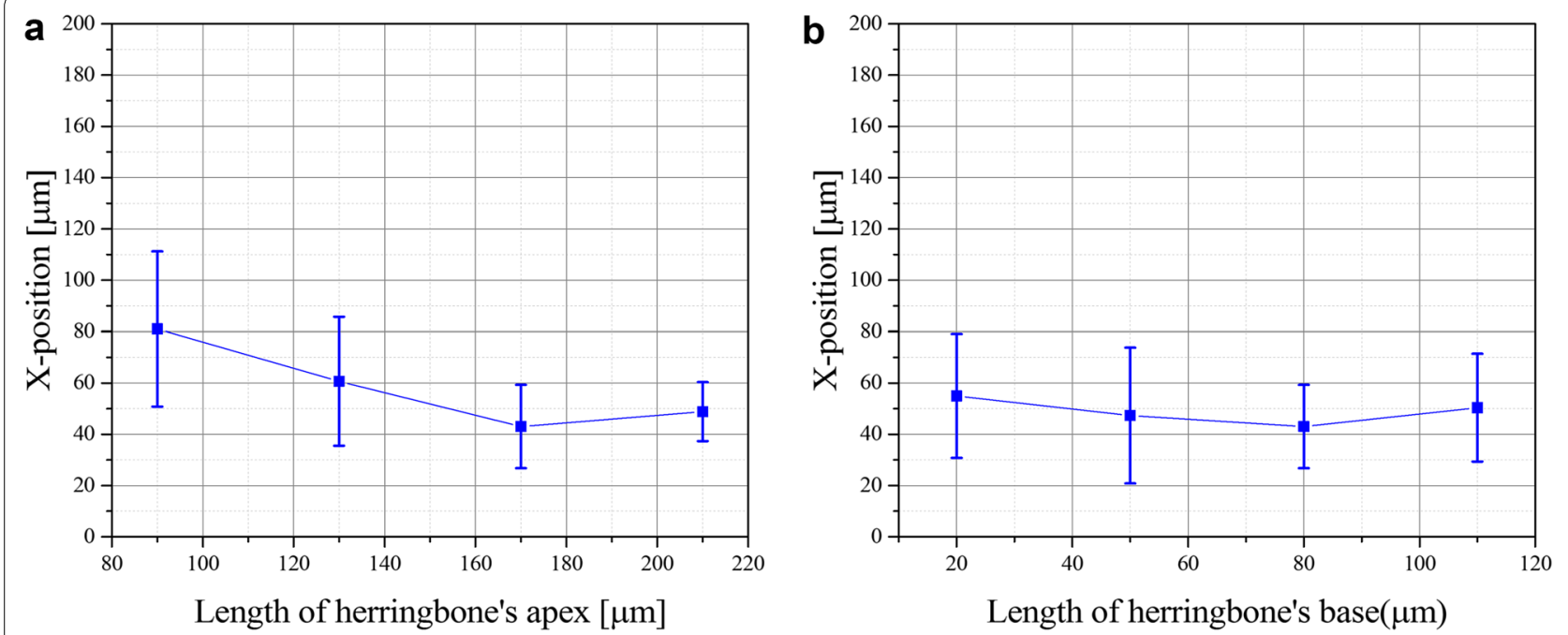

Fig. 4 The result of particle positioning across the channel. $\mathbf{a} X$ axis and $Y$ axis represents length of the apex and position across the channel. $\mathbf{b} X$ axis and $Y$ axis represents length of the base and position across the channel 


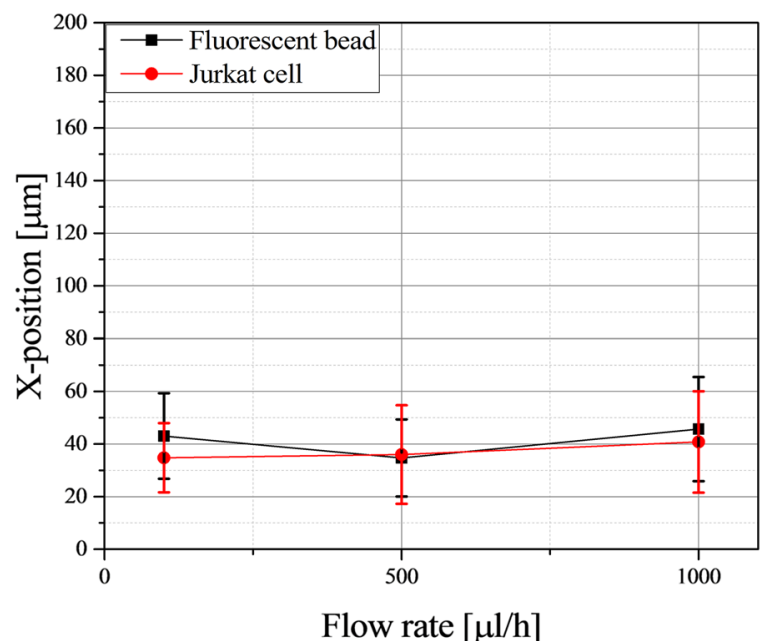

Fig. 6 Particles' and cells' position along the channel at different flow rates. The $X$ axis and $Y$ axis represent flow rate and $x$-position across the channel

of the focused particle stream and its density. By adjusting these parameters, cell positioning closer to herringbones' apex can be achieved.

\section{Application of the device to cell trapping}

The herringbone structure, designed and further improved herein, was tested in conjunction with an array of trapping structures with a goal to enhance trapping efficiency $[8,9]$. The following equation represents trapping efficiency Eq. 1.

Trapping efficiency (\%)

$$
=\frac{\text { Number of trapped cells }}{\text { Total number of trapping structures }} \times 100
$$

As shown in Fig. 7a, the whole device consists of two regions with distinct functions, i.e. series of herringbone structures and trapping structures. The trapping structure region contained 100 individual structures. It was designed by using flow rate ratio, i.e. the flow rate ratio determines which streamlines flow into the trapping structure. The cells tend to follow their current streamlines. The flow rate ratio between the main channel and the trapping structure can be determined from the fluidic resistance, $\Delta P=Q R$. From the equation, assuming $\Delta P$ is constant, when the resistance $(R)$ increases, the flow $(Q)$ is reduced. Thus, the flow can be regulated by changing the fluidic resistance, which in its turn modulates the critical diameter of streamlines that flow through the trapping structure. For calculating flow resistance of the channel, the fluidic channel can be considered analogous to an electrical circuit. Ohm's law, $\Delta V=I R$ can be used to represent $\Delta P=Q R$. In an electrical circuit, the $I$ (electrical current) denotes the flow $(Q)$, the resistance denotes the length of fluid channel $(R)$ [7]. The fluid resistance is adjusted by changing length of the through-hole channel in the trapping structure, represented by $x$ in Fig. 7. The desired length of $x$ was analytically calculated. For confirming the flow rate ratio, a numerical study was carried out using COMSOL 5.2 simulation software.

The proposed device's performance was compared against a device without herringbone structures. The experimental testing was conducted using a solution with concentration of cells at $1.7 \times 10^{5}$ cells $/ \mathrm{ml}$, which was introduced into the device at $100 \mu \mathrm{l} / \mathrm{h}$ flow rate. Cells were labeled with calcein AM (Invitrogen, USA). The device that had no herringbone structures didn't affect the positioning of cells across the channel and the trapping efficiency was recorded to be around $40 \%$. Random distribution of cells in the flow decreases their chance to follow streamlines that enter trapping structures, thus the low trapping efficiency was observed. On the other hand, the device with herringbone structures has demonstrated approximately $86 \%$ trapping efficiency. The comparison of trapping efficiencies of both devices is presented in Fig. 8.

\section{Conclusion}

In this study, a microfluidic device containing a series of continuously varying herringbone structures was demonstrated to be effective in manipulation of cells' position 

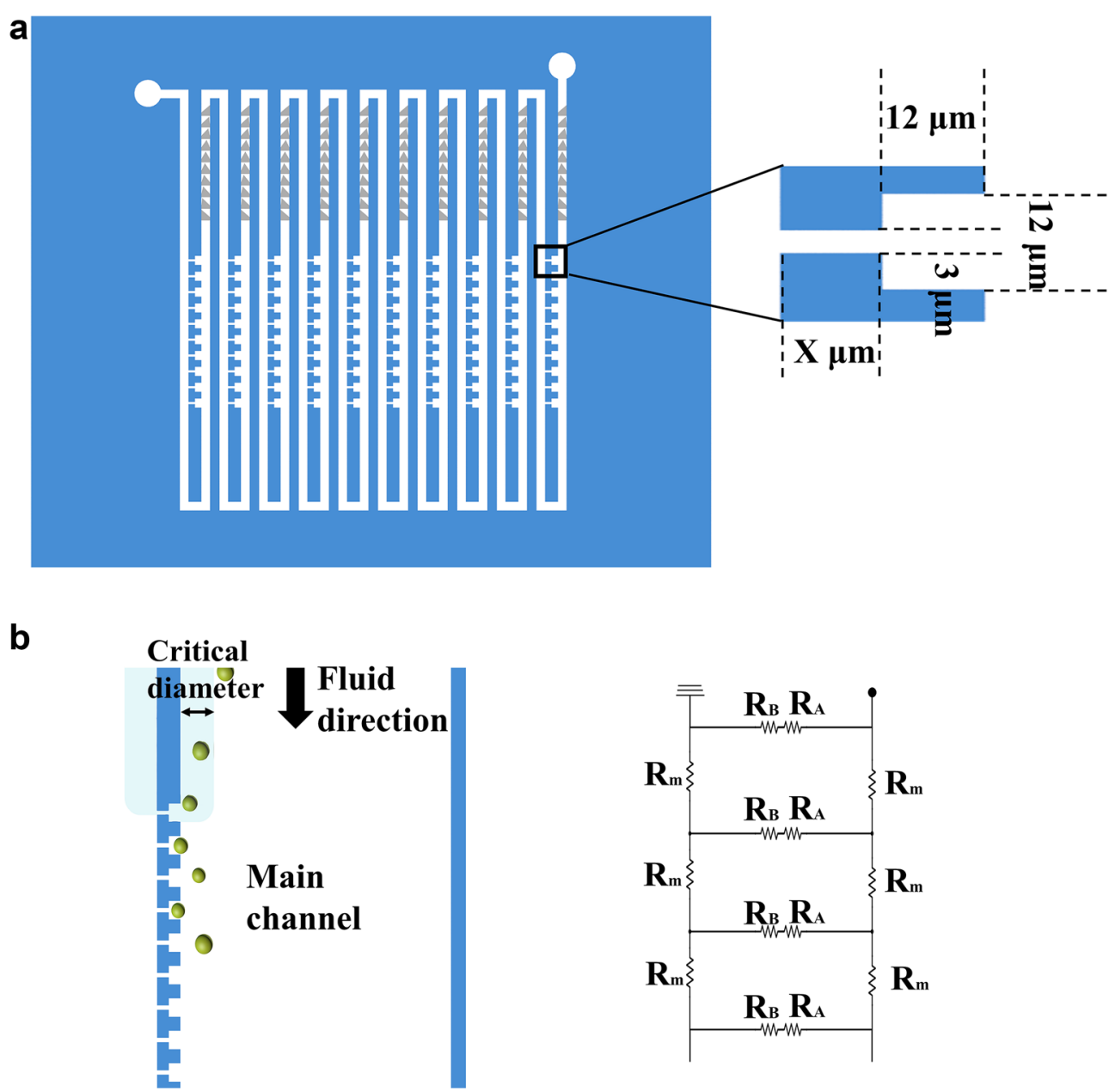

Fig. 7 Schematic representation of the microfluidic device with a cell trapping region. a The device consists of series of herringbone structures and trapping structures. The main channel's width is $200 \mu \mathrm{m}$ and the number of trapping structures is 100 . b Schematic diagram of the trapping structure (left) and analogous electrical circuit representing electrical resistance. RA, RB: Fluidic resistance of the trapping structure, Rm: fluidic resistance of the main channel, the ground sign is the outlet, dot sign is the inlet

without application of external forces. It has been determined that the length of herringbones' apex $(170 \mu \mathrm{m})$ and the length ratio between the flat channel and the herringbone structure (1:1.5) were important variables affecting the cells' $x$-position along the channel. On the contrary, the length of herringbones' base didn't have a significant effect on the $x$-position. One of the possible applications of such device was also demonstrated, i.e. 

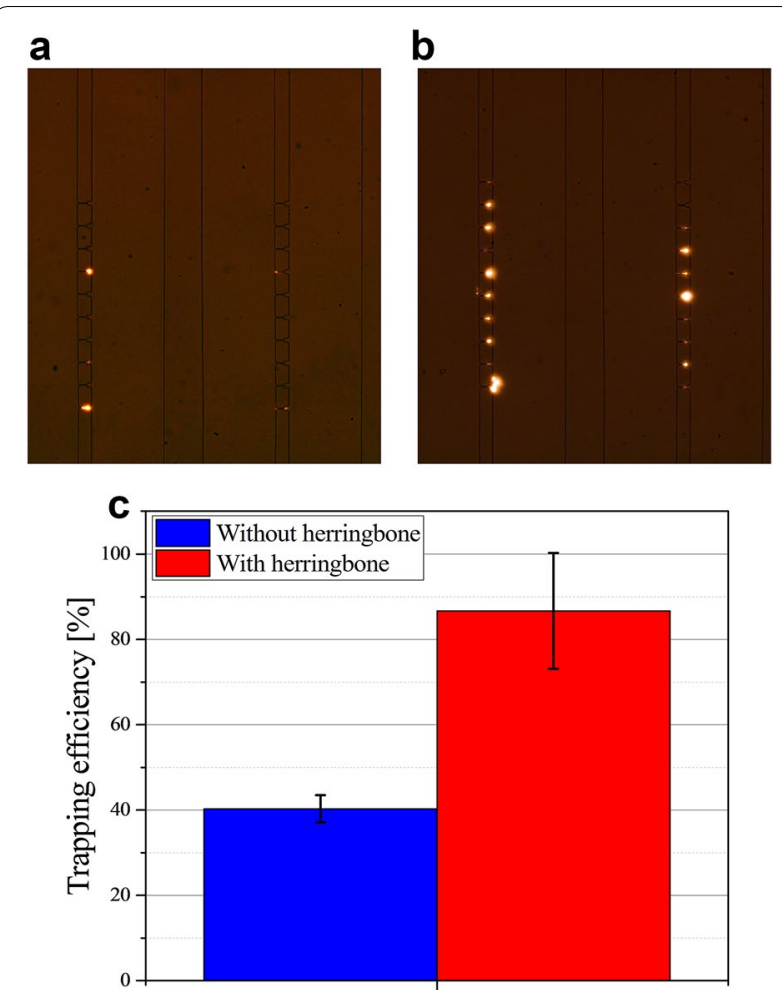

Fig. 8 Demonstration devices' efficiency with and without herringbone structures. Devices had 100 trapping structures. a The trapping structures in 7th and 8th lines without herringbone structures. $\mathbf{b}$ The trapping structures in 7 th and 8 th lines with herringbone structures. c A graph comparing trapping efficiency

the proposed device was used to improve cell trapping efficiency. Cell positioning in a microfluidic system has a promising prospect to be utilized for cellular analysis in the field of cell biology.

\section{Authors' contributions}

YGJ has designed and fabricated the device, also carried out the experimental study and drafted the manuscript as a first author. JCH, JC discussed and advised on the design and fabrication process. AA provided assistance during fabrication and drafting of the manuscript. SY supervised the project and completed the manuscript. All authors read and approved the final manuscript.

\section{Author details}

${ }^{1}$ Department of Biomedical Science and Engineering, Gwangju Institute of Science and Technology (GIST), Gwangju 61005, Republic of Korea. ${ }^{2}$ School of Mechanical Engineering, Gwangju Institute of Science and Technology (GIST), Gwangju 61005, Republic of Korea.

\section{Competing interests}

The authors declare that they have no competing interests.

\section{Funding}

This material is based upon work supported by the Ministry of Trade, Industry and Energy (MOTIE, Korea) under Industrial Technology Innovation Program No. 10062533, 'Development of a microfluidic-based multiplex (6 items) point of care chip for differential diagnosis between bacterial and viral upper respiratory infection using blood within $20 \mathrm{~min}$ and the National Research Foundation of Korea (NRF) Grant funded by the Korea government (MSIP) (NRF-2016M3A7B4910556).

Received: 15 December 2016 Accepted: 31 December 2016

Published online: 09 January 2017

\section{References}

1. Yun H, Kim K, Lee WG (2013) Cell manipulation in microfluidics. Biofabrication 5(2):022001

2. Manbachi A, Shrivastava S, Cioffi M, Chung BG, Moretti M, Demirci U et al (2008) Microcirculation within grooved substrates regulates cell positioning and cell docking inside microfluidic channels. Lab Chip 8(5):747-754

3. Frenea-Robin M, Haddour N (2009) On-chip cell positioning and sorting using contactless methods: a comparision between different force-fields. Biomed Eng pp 41-46

4. Rhee SW, Taylor AM, Cribbs DH, Cotman CW, Jeon NL (2007) External force-assisted cell positioning inside microfluidic devices. Biomed Microdevices 9(1):15-23

5. Kim J, Erath J, Rodriguez A, Yang C (2014) A high-efficiency microfluidic device for size-selective trapping and sorting. Lab Chip 14(14):2480-2490

6. McGrath J, Jimenez M, Bridle H (2014) Deterministic lateral displacement for particle separation: a review. Lab Chip 14(21):4139-4158

7. Yang S, Ündar A, Zahn JD (2005) Blood plasma separation in microfluidic channels using flow rate control. ASAIO J 51(5):585-590

8. Iwai K, Tan WH, Ishihara H, Takeuchi S (2011) A resettable dynamic microarray device. Biomed Microdevices 13(6):1089-1094

9. Kim H, Lee S, Lee JH, Kim J (2015) Integration of a microfluidic chip with a size-based cell bandpass filter for reliable isolation of single cells. Lab Chip 15(21):4128-4132

\section{Submit your manuscript to a SpringerOpen ${ }^{\circ}$ journal and benefit from:}

- Convenient online submission

- Rigorous peer review

- Immediate publication on acceptance

- Open access: articles freely available online

- High visibility within the field

- Retaining the copyright to your article

Submit your next manuscript at springeropen.com 